\title{
A Method for the Numerical Integration of Ordinary Differential Equations
}

\section{by L. Stoller and D. Morrison}

1. Introduction. We consider the problem of solving numerically a differential equation $y^{\prime}=f(x, y)$ with initial condition $y\left(x_{0}\right)=y_{0}$. Our point of departure is the formula

$$
y\left(x_{0}+h\right)=y\left(x_{0}\right)+\int_{x_{0}}^{x_{0}+h} f[x, y(x)] d x,
$$

where $y(x)$ denotes the solution of the differential equation. The idea is to use a quadrature formula to estimate the integral of (1). This requires knowledge of the integrand at specified arguments $x_{i}$ in $\left(x_{0}, x_{0}+h\right)$-hence we require the values of $y(x)$ at these arguments. A numerical integration method may be used to estimate $y(x)$ for the required arguments. In this way a numerical integration method is combined with a quadrature formula to obtain another numerical integration method.

A large number of methods may be devised, depending on which combination of quadrature formula and integration method is used. In particular, the Gauss two-point quadrature formula combined with the Runge-Kutta fourth order method appears to give excellent results [1]. We propose here the combination of the Radau three-point quadrature formula with the Runge-Kutta fourth order method. The resulting method seems to give greater accuracy with the same amount of work.

2. The Method. The Radau quadrature formula [2] gives

$$
y\left(x_{0}+h\right)=y\left(x_{0}\right)+\frac{h}{2}\left[W_{0} y^{\prime}\left(x_{0}\right)+W_{\mathbf{q}} y^{\prime}\left(x_{q}\right)+W_{p} y^{\prime}\left(x_{p}\right)\right]-\epsilon_{Q},
$$

where

$$
\begin{array}{ll}
W_{0}=\frac{2}{9} & \\
W_{q}=\left(\frac{8}{9}+\frac{\sqrt{6}}{18}\right) & x_{q}=x_{0}+h\left(\frac{3}{5}-\frac{\sqrt{6}}{10}\right)=x_{0}+h q \\
W_{p}=\left(\frac{8}{9}-\frac{\sqrt{6}}{18}\right) & x_{p}=x_{0}+h\left(\frac{3}{5}+\frac{\sqrt{6}}{10}\right)=x_{0}+h p
\end{array}
$$

and

$$
\epsilon_{Q}=\frac{-h^{6}}{72,000} \frac{d^{6} y(\eta)}{d x^{6}}, \quad x_{0}<\eta<x_{0}+h .
$$

The method proceeds as follows: We use the Runge-Kutta fourth order method to integrate from $x_{0}$ to $x_{q}$ and then from $x_{q}$ to $x_{p}$, thus obtaining estimates $y_{q}$ and $y_{p}$

Received 7 March 1958. 
for $y\left(x_{q}\right)$ and $y\left(x_{p}\right)$. Then formula (2) is used to obtain an estimate $y_{k}$ of $y\left(x_{0}+h\right)$ :

$$
y_{h}=y\left(x_{0}\right)+\frac{h}{2}\left[W_{0} f\left(x_{0}, y_{0}\right)+W_{q} f\left(x_{q}, y_{q}\right)+W_{p} f\left(x_{p}, y_{p}\right)\right]
$$

3. The Error. We shall be concerned in this section with a description of the local truncation error of the method described in section 2. The local truncation error of a single step numerical integration method is defined as follows. Let $y(x)$ denote an exact solution of the equation $y^{\prime}=f(x, y)$ with initial condition $y\left(x_{0}\right)=y_{0}$. Let $y_{h}$ denote the approximation to $y\left(x_{0}+h\right)$ obtained by the use of one step of the numerical method. Then the local truncation error $R\left(x_{0}, y_{0}, h\right)^{\prime}$ is defined by

$$
R\left(x_{0}, y_{0}, h\right)=y_{h}-y\left(x_{0}+h\right) .
$$

The local truncation error of the Runge-Kutta method will be denoted by $R_{1}\left(x_{0}, y_{0}, h\right)$, and may be written in the form:

$$
R_{1}\left(x_{0}, y_{0}, h\right)=\phi\left(x_{0}, y_{0}\right) h^{5}+0\left(h^{6}\right) .
$$

If $y_{1}(x)$ denotes the exact solution of the differential equation which satisfies the initial condition $y_{1}\left(x_{q}\right)=y_{q}$ then the errors at $x_{q}$ and $x_{p}$ may be written

$$
\begin{aligned}
y_{q}-y\left(x_{q}\right) & =\phi\left(x_{0}, y_{0}\right)(q h)^{5}+0\left(h^{6}\right) \\
y_{p}-y_{1}\left(x_{p}\right) & =\phi\left(x_{q}, y_{q}\right)(p-q)^{5} h^{5}+0\left(h^{6}\right) .
\end{aligned}
$$

Assuming the function $\phi$ is sufficiently smooth, we may replace $\phi\left(x_{q}, y_{q}\right)$ in (7) by $\phi=\phi\left(x_{0}, y_{0}\right)$ since this only affects the $0\left(h^{6}\right)$ term. The function $\eta(x)=$ $y_{1}(x)-y(x)$ satisfies the differential equation

$$
\eta^{\prime}(x)=f\left(x, y_{1}\right)-f(x, y) .
$$

An application of the mean value theorem gives

$$
\eta^{\prime}(x)=f_{y}(x, \xi) \eta(x)
$$

where $\xi=\xi(x)$ is a number between $y_{1}(x)$ and $y(x)$. It follows that

$$
\begin{aligned}
\eta\left(x_{p}\right) & =\eta\left(x_{q}\right)+\eta^{\prime}\left(x_{q}\right)\left(x_{p}-x_{q}\right)+\cdots \\
& =\eta\left(x_{q}\right)(1+0(h)) .
\end{aligned}
$$

But by (6) $\eta\left(x_{q}\right)=\phi(q h)^{5}+0\left(h^{6}\right)$. Using the definition of $\eta(x)$ we have:

$$
y_{1}\left(x_{p}\right)-y\left(x_{p}\right)=\phi(q h)^{5}+0\left(h^{6}\right) \text {. }
$$

Now, combining (7) and (11)

$$
y_{p}-y\left(x_{p}\right)=\phi(q h)^{5}+\phi(p-q)^{5} h^{5}+0\left(h^{6}\right) .
$$

The error in the derivatives may now be computed:

$$
\begin{aligned}
& f\left(x_{q}, y_{q}\right)-f\left(x_{q}, y\left(x_{q}\right)\right)=f_{y} \phi(q h)^{5}+0\left(h^{6}\right) \\
& f\left(x_{p}, y_{p}\right)-f\left(x_{p}, y\left(x_{p}\right)\right)=f_{y}\left[\phi(q h)^{5}+\phi(p-q)^{5} h^{5}\right]+0\left(h^{6}\right)
\end{aligned}
$$

where we have again used the mean value theorem. We have also assumed that $f_{y}(x, y)$ is constant and put the resulting error into the $0\left(h^{6}\right)$ term. We may now 


\section{TABLE I}

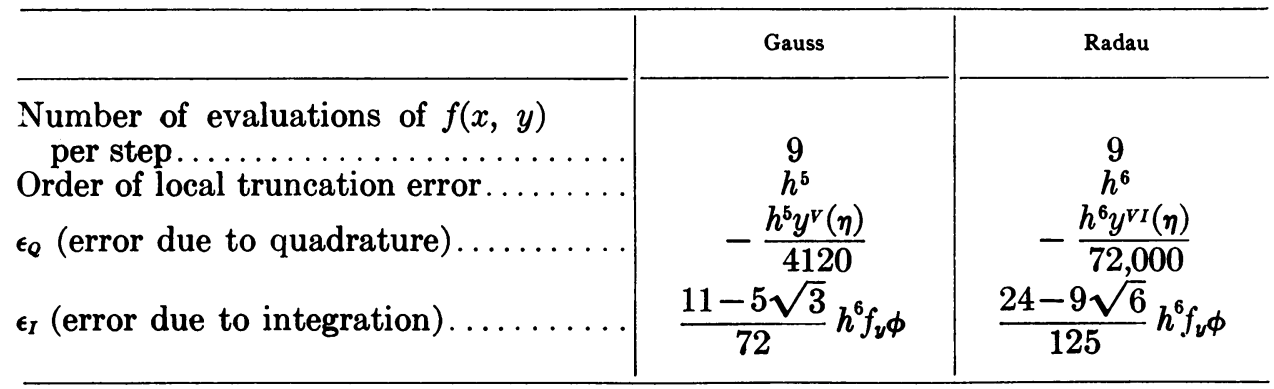

\section{TABLE II}

\begin{tabular}{c|l|c|c}
\hline \multirow{2}{*}{ Problem } & \multicolumn{1}{|c|}{ Method } & $\begin{array}{c}\text { Evaluations } \\
\text { of } f(x, y)\end{array}$ & Error at $x=1$ \\
\cline { 2 - 3 } (a) & Gauss & 36 & $2.74 \times 10^{-6}$ \\
& Radau & 36 & $0.298 \times 10^{-6}$ \\
(b) & Runge-Kutta & 64 & $0.298 \times 10^{-6}$ \\
(c) & Gauss & 144 & $0.229 \times 10^{-4}$ \\
& Radau & 144 & $0.0977 \times 10^{-4}$ \\
& Runge-Kutta & 128 & $0.815 \times 10^{-3}$ \\
& Gauss & 126 & $0.282 \times 10^{-3}$ \\
& Radau & 126 & $0.116 \times 10^{-3}$ \\
\hline
\end{tabular}

compute the local truncation error $R$ of the method of section 2. From (2), (3), (13) and (14) we obtain

$$
\begin{aligned}
R\left(x_{0}, y_{0}, h\right) & =y_{h}-y\left(x_{0}+h\right) \\
& =\frac{h}{2}\left\{W_{q} f_{y} \phi(q h)^{5}+W_{p} f_{\nu} \phi\left[(q h)^{5}+(p-q)^{5} h^{5}\right]\right\}+\epsilon_{Q}+0\left(h^{6}\right) \\
& =\epsilon_{I}+\epsilon_{Q}+0\left(h^{6}\right) .
\end{aligned}
$$

A similar analysis may be performed on the method which combines Gauss quadrature with Runge-Kutta integration, and Table I summarizes the results. Table II gives some of the numerical results which were obtained on the 1103A. Also included in Table II is a comparison with results obtained from the use of the Runge-Kutta formula alone.

\section{Problems}

(a) $f(x, y)=y, x_{0}=0, y_{0}=1$. Solution: $y=e^{x}$

(b) $f(x, y)=\frac{5 y}{1+x}, x_{0}=0, y_{0}=1$. Solution: $y=(1+x)^{5}$

(c) $f(x, y)=\frac{6 y}{1+x}, x_{0}=0, y_{0}=1$. Solution: $y=(1+x)^{6}$

Space Technology Laboraties, Inc.

Los Angeles, California

1. P. HenRICI, Lecture notes on the numerical solution of ordinary differential equations (UCLA). 
2. F. B. Hildebrand, Introduction to Numerical Analysis, McGraw-Hill, New York, 1956.

3. H. Flat \& S. Conte, Integration of Ordinary Differential Equations, NN-21, August 30, 1956, The Ramo-Wooldridge Corporation, Los Angeles, California.

\section{Numerical Evaluation of Multiple Integrals II}

\section{by Preston C. Hammer and Arthur H. Stroud}

1. Introduction. In the first paper of this title [1], Hammer and Wymore introduced methods whereby integration formulas of the form

$$
\int_{R} w(x) f(x) d x \doteq \sum a_{i} f\left(\xi_{i}\right)
$$

which are known for special regions (in $n$-dimensional euclidean space $E_{n}$ ) may be used to determine formulas for other regions. They also showed, in some cases, how the symmetry of a region may simplify the task of finding integration formulas for the region.

To facilitate numerical integration over regions in higher dimensional spaces, we summarize the most important formulas (1) and review methods by which formulas for classes of regions may be obtained from them. These methods enable one to obtain formulas for regions which we consider too special to warrant particular formulas.

2. Regions with symmetry. In deriving numerical integration formulas it is possible to obtain explicit formulas with comparatively little effort when the region and the formula both are assumed to have certain kinds of symmetry. Formulas precise for polynomial functions involving minimal numbers of points can be derived, in principle, by solving simultaneous algebraic equations by general elimination procedures leading to polynomials which have as roots the solutions of the system. However, the manipulative work in achieving such solutions is forbidding in magnitude and can probably be done effectively only with high speed computers. Moreover, the solutions may turn out to be complex numbers and the determinations of approximate values for the solutions will involve the numerical solution of high degree polynomial equations. For example, the general seventh degree polynomial in three variables has 120 terms so that the determination of a numerical integration formula precise for all such polynomials over an arbitrary region $R$ would lead to the problem of solving a system of 120 equations of algebraic (non-linear) character. In [1] it is shown that for certain symmetrical regions with a symmetrical formula the problem is reduced to seven simultaneous algebraic equations for which explicit solutions are easily derived. The problem of finding integrals of monomials to establish the equations to be solved also will be a difficult problem for many regions.

A set $S$ in $E_{n}$ is said to be fully symmetrical provided $x \in S$ implies that every

Received 24 March 1958. This work is supported in part by the Office of Ordnance Research, U. S. Army contract no. DA-11-022-ORD-2301, and in part by the Graduate Research Committee of the University of Wisconsin. 